\title{
Intrinsic Role of Polysialylated Neural Cell Adhesion Molecule in Photic Phase Resetting of the Mammalian Circadian Clock
}

\author{
Rebecca A. Prosser, ${ }^{1}$ Urs Rutishauser, ${ }^{2}$ Grace Ungers, ${ }^{2}$ Lenka Fedorkova, ${ }^{3}$ and J. David Glass ${ }^{3}$ \\ ${ }^{1}$ Department of Biochemistry and Cellular and Molecular Biology, University of Tennessee, Knoxville, Tennessee 37996, ${ }^{2}$ Program in Cellular Biochemistry \\ and Biophysics, Memorial Sloan-Kettering Cancer Center, New York, New York 10021, and ${ }^{3}$ Department of Biological Sciences, Kent State University, Kent, \\ Ohio 44242
}

The suprachiasmatic nuclei (SCN), the location of the mammalian circadian clock, are one of the few adult brain regions that express the highly polysialylated form of neural cell adhesion molecule (PSA-NCAM). A role for the polysialic acid (PSA) component of PSA-NCAM, which is known to promote tissue plasticity, has been reported for photic entrainment of circadian rhythmicity in vivo. The in vivo results, however, do not discriminate between PSA acting upstream or downstream of the glutamatergic synapses that convey photic information to the SCN. To address this key issue, we exploited an in vitro rat brain slice preparation that retains robust circadian function. As in the intact SCN, PSA levels in the isolated SCN are rhythmic, with higher levels during the early subjective day and lower levels during subjective night. Importantly, bath application of glutamate to SCN slices rapidly and transiently increases PSA levels during both the subjective day and night. Pretreating the slices with endoneuraminidase, which selectively removes PSA from NCAM and thereby prevents this increase, abolishes glutamate- and optic chiasm stimulation-induced phase delays of the SCN circadian neuronal activity rhythm. These results support the hypothesis that PSA expression in the SCN is controlled by both the circadian clock and photic input to the clock and that expression of PSA in the SCN is critical for photic-like phase shifts of the clock. Together, these results establish that such actions of PSA are manifested downstream from presynaptic retinohypothalamic terminals and therefore are intrinsic to the SCN itself.

Key words: circadian rhythms; suprachiasmatic nucleus; polysialic acid; NCAM; phase-shift; glutamate; endoneuraminidase

\section{Introduction}

The mammalian suprachiasmatic nuclei (SCN) contain an endogenous circadian clock (Zlomanczuk and Schwartz, 1999). The SCN clock maintains synchrony with the external environment primarily through light input, such that light pulses presented during early night delay the clock, whereas light pulses presented during late night advance the clock. The cellular processes underlying photic phase shifts are complex and incompletely understood but are thought to be initiated by glutamate release from retinohypothalamic (RHT) terminals in the SCN, resulting in postsynaptic increases in intracellular $\mathrm{Ca}^{2+}$ and, ultimately, an increased transcription of genes associated with the cellular clock mechanism (Gillette and Tischkau, 1999).

The SCN express a highly polysialylated form of neural cell adhesion molecule (PSA-NCAM) (Glass et al., 1994; Shen et al., 1997, 1999). PSA-NCAM is a negative regulator of cell-cell interactions mediated by a wide variety of receptors (Fujimoto et al., 2001), and this action facilitates important developmental events associated with cell migration and axon guidance (Rutishauser and Landmesser, 1996). Whereas levels of NCAM polysialylation are high during development and generally low in

Received June 17, 2002; revised 0ct. 28, 2002; accepted Nov. 1, 2002.

This work was supported by National Institutes of Health Grants MH53317 (R.A.P.), HD 18369 (U.R.), and MH57034 (J.D.G.).

Correspondence should be addressed to Rebecca A. Prosser at the above address. E-mail: rprosser@utk.edu. Copyright $\odot 2003$ Society for Neuroscience $\quad 0270-6474 / 03 / 220652-07 \$ 15.00 / 0$ adult tissues (Chuong and Edelman, 1984; Seki and Arai, 1993), PSA-NCAM continues to be expressed in adult brain regions that have the capacity for structural and/or physiological plasticity. Such regions include the olfactory bulb (Miragall et al., 1990), hippocampus-dentate gyrus (Seki and Arai, 1991, 1993; Le Gal La Salle et al., 1992; Becker et al., 1996; Muller et al., 1996), and hypothalamus (Murakami et al., 1991; Theodosis et al., 1991; Bonfanti et al., 1992; Glass et al., 1994; Lee et al., 1995; Viguie et al., 2001). Evidence for a functional role of polysialic acid (PSA) in brain physiology is suggested by the observations that removing PSA produces ectopic synaptic-like structures (Seki and Rutishauser, 1998), abolishes long-term potentiation (LTP) in the hippocampus (Muller et al., 1996), and prevents structural glialneuronal reorganizations of the supraoptic nucleus induced by lactation and dehydration (Theodosis et al., 1999).

The expression of PSA-NCAM in the SCN suggests that plasticity in cell interactions is also important to circadian timekeeping regulation. This contention is supported by initial in vivo findings that removing PSA impairs circadian rhythm stability (Shen et al., 1997, 2001) and attenuates photic signaling events in the SCN (Glass et al., 2000b). However, in vivo it has not been possible to discriminate between effects that PSA might have on intrinsic SCN functions as opposed to events that are involved in providing input to the SCN from the retina. For this purpose, we adopted and validated the in vitro rat SCN slice preparation (Gillette et al., 1995) for the study of PSA in circadian photic entrainment in the isolated SCN. Having direct access to the isolated 
SCN permits a more selective activation of SCN photic responses, namely by glutamate and by optic chiasm stimulation. The demonstration that these more central perturbations remain highly dependent on PSA supports the conclusion that PSA is essential for intrinsic aspects of clock entrainment.

\section{Materials and Methods \\ Brain slice preparation}

Coronal brain slices $(500 \mu \mathrm{m})$ containing the SCN were prepared during the daytime from adult, male Sprague Dawley rats housed in a $12 \mathrm{hr}$ light/dark cycle as reported previously (Prosser and Gillette, 1989; Prosser et al., 1993, 1998b). Slices were maintained at the interface of a Hatton-style brain slice chamber (Hatton et al., 1980), in which they were perfused continuously with warm $\left(37^{\circ} \mathrm{C}\right)$, oxygenated $\left(95 \% \mathrm{O}_{2}-5 \%\right.$ $\mathrm{CO}_{2}$ ), glucose-bicarbonate-supplemented Earle's balanced salt solution (Sigma, St. Louis, MO), pH 7.4.

\section{Single-unit recordings and data analysis}

Single-unit recordings were obtained using methods described previously (Prosser et al., 1993; Prosser, 1998b). Briefly, the spontaneous activity of single SCN neurons was recorded using glass capillary microelectrodes filled with $3 \mathrm{M} \mathrm{NaCl}$. Each neuron was recorded for $5 \mathrm{~min}$, and the data were stored for later determination of firing rate using a DataWave (Longmont, CO) system. Typically, four to seven cells were recorded during each hour. These firing rates were then used to calculate 2 $\mathrm{hr}$ running averages, lagged by $1 \mathrm{hr}$, to obtain a measure of population neuronal activity. As in previous studies (Prosser et al., 1993; Prosser, 1998b), the time of peak neuronal activity was assessed visually by estimating, to the nearest $15 \mathrm{~min}$, the time of symmetrically highest activity.

\section{Experimental protocols}

Drug treatments. Glutamate (1 $\mathrm{mm})$ was bath applied to the brain slices beginning at either zeitgeber time 6 (ZT 6) (ZT 0 is lights-on in the donor colony) or ZT 14 on the first day in vitro by stopping the perfusion and replacing the medium in the slice chamber with medium containing glutamate. After $10 \mathrm{~min}$, the normal medium was reintroduced into the slice chamber, and perfusion was resumed. Another group of slices was treated in a similar manner at ZT 6 with a combination of glutamate antagonists. For blocking experiments using endoneuraminidase (endo $\mathrm{N}$ ), at ZT 12 on the first day in vitro, the bathing medium was replaced with medium containing endo $\mathrm{N}$ diluted to $20-100 \mathrm{U} / \mathrm{ml}$. The endo $\mathrm{N}$-containing medium (total volume, $15 \mathrm{ml}$; chamber volume, $<3 \mathrm{ml}$ ) was continually recirculated between the slice chamber and a reservoir in which it was rewarmed and reoxygenated. After $2 \mathrm{hr}$, the recirculation was stopped, and the medium in the slice chamber was replaced with medium containing endo $\mathrm{N}$ and glutamate. After $10 \mathrm{~min}$, this medium was exchanged for medium containing endo $\mathrm{N}$, and the recirculating perfusion was reinstated. After 50 additional minutes, this medium was replaced with normal medium, and normal perfusion was resumed. Control experiments were conducted to determine whether the recirculation procedure (using normal medium) altered the circadian rhythm of neuronal activity. No effect was seen (data not shown). Therefore, the times-of-peak for drug-treated slices were compared with the mean time-of-peak for untreated slices (ZT $6.0 \pm 0.3 ; n=3$ ) to determine the amount of phase shift induced by the treatment. Chemicals used in the study were L-glutamate, 6-cyano-7-nitroquinoxaline-2,3-dione (CNQX), 2-amino-5-phosphonopentanoic acid (AP-5) (all from Sig$\mathrm{ma}$ ), and endo N (U. Rutishauser).

Optic chiasm stimulation. Optic chiasm stimulation was performed as described previously (Prosser, 1998a). Briefly, a bipolar, blunt-cut, insulated platinum-iridium electrode was positioned in the optic chiasm ventrolateral to the $\mathrm{SCN}$. Voltage $(10 \mathrm{~Hz}, 10 \mathrm{~V}, 3 \mathrm{msec}$ duration) was applied for $10 \mathrm{~min}$.

Daily rhythm of PSA expression. To determine whether a daily rhythm in PSA expression persists in vitro, SCN brain slices were prepared during the subjective day and maintained as described above. Slices were then removed from the slice chamber at 4 different time points (ZT 6, ZT 12, ZT 18, and ZT 24), and the optic chiasm was removed. The remaining tissue, consisting almost entirely of SCN tissue, was frozen on dry ice.
Samples consisting of single SCN slices were then stored at $-80^{\circ} \mathrm{C}$. Western blot analyses of tissue PSA content were subsequently performed on the tissue extracts as described below.

Immunological analyses

Immunoblot analyses. Brain slices were prepared as described above, trimmed to contain only the SCN and underlying optic chiasm. Time of slice preparation was varied to equalize the time in vitro as much as possible. Slices were maintained in the slice chamber for at least $1 \mathrm{hr}$ after slice preparation. At the appropriate time, each slice was removed and rapidly frozen on dry ice, and the optic chiasm was removed using a single-edged razor blade. The remaining tissue was then weighed and stored at $-80^{\circ} \mathrm{C}$. The samples were weighed and sonicated in $20 \mathrm{vol}$ of 0.01 м PBS, pH 7.4, containing Nonidet P-40 (Sigma) and aprotinin (20 $\mu \mathrm{g} / \mathrm{ml}$; Sigma). The tissue homogenates were centrifuged at $13,600 \times g$ at $4^{\circ} \mathrm{C}$ for $5 \mathrm{~min}$. A $1 \mu \mathrm{l}$ aliquot of the supernatant from each sample was used for protein determination using a micro BCA protein assay reagent kit (Pierce, Rockford, IL). The remainder of the supernatant was diluted with 1 vol of $1.6 \mathrm{ml}$ of glycerol, $3.0 \mathrm{ml}$ of $10 \%$ SDS, $0.8 \mathrm{ml}$ of 2,6mercaptoethanol, and $0.4 \mathrm{ml}$ of $0.05 \%$ bromophenol blue, boiled for 3 min, and stored at $-70^{\circ} \mathrm{C}$ until immunoblot analysis. An equal amount of total protein $(20 \mu \mathrm{g})$ from each sample was loaded on a 5\% SDSpolyacrylamide gel. The protein was transferred to nitrocellulose membrane (Hybond-ECL; Amersham Biosciences, Arlington Heights, IL) at $35 \mathrm{~V}$ for $18 \mathrm{hr}$, and PSA was detected by incubation with a characterized mouse monoclonal antibody 5A5 (IgM) against $\alpha$-2-8-linked PSA of NCAM (Acheson et al., 1991), followed by a peroxidase-conjugated second antibody, and then reacting the bound peroxidase with chemiluminescent substrate (ECL; Amersham Biosciences). Chemiluminescence was detected by exposure to x-ray film (Hyperfilm-ECL; Amersham Biosciences). The exposed signals on the film were scanned and then quantified using Gel-Pro software (Media Cybernetics, Silver Spring, MD).

Immunohistochemical procedures. Brain slices were prepared and maintained for at least $1 \mathrm{hr}$ after slice preparation. At the appropriate time, each slice was removed from the slice chamber and placed in $2 \%$ paraformaldehyde for $3 \mathrm{hr}$ at $4^{\circ} \mathrm{C}$. The $500-\mu \mathrm{m}$-thick slices were then placed in $0.1 \mathrm{M} \mathrm{PBS}$ and kept at $4^{\circ} \mathrm{C}$. Subsequently, the slices were glued to a cutting block with cyanoacrylate, and vibratome sections ( $60 \mu \mathrm{m}$ thick) were cut, washed in $0.1 \%$ PBS, and incubated with $1 \% \mathrm{H}_{2} \mathrm{O}_{2}$ to inactivate endogenous peroxidase. Sections then were sequentially incubated in the following solutions with buffer washes in between: (1) 5A5 primary antibody diluted 1:500 with incubation buffer $(50.0 \mathrm{~mm}$ Tris-buffered saline, $\mathrm{pH} 7.6$, containing $0.9 \% \mathrm{NaCl}, 0.1 \%$ bovine serum albumin, and $0.1 \%$ Triton X-100) at $4^{\circ} \mathrm{C}$ overnight; (2) secondary antibody conjugated with peroxidase (goat anti-mouse IgM diluted 1:200; Vector Laboratories, Burlingame, CA) at room temperature for $1 \mathrm{hr}$; and (3) $0.02 \%$ diaminobenzidine with $0.009 \% \mathrm{H}_{2} \mathrm{O}_{2}$ at room temperature for 5-10 $\mathrm{min}$. Stained sections were floated on to a gel-coated slide and mounted with coverslips with Permount (Fisher Scientific, Houston, TX).

\section{endo $N$ preparation}

Recombinate endo $\mathrm{N}$ was expressed by isopropylthioglucose induction of pREP4-repressed M15 cells containing a QE60 (catalog \#33603; Qiagen, Hilden, Germany) plasmid encoding endo N (Vimr et al., 1984) plus a 6 xHis-tag. After $3 \mathrm{hr}$, the cell pellet was lysed in $50 \mathrm{~mm} \mathrm{NaH}_{2} \mathrm{PO}_{4}, \mathrm{pH}$ 8.0, $300 \mathrm{~mm} \mathrm{NaCl}$, and $10 \mathrm{~mm}$ imidiazole plus !X Protease Inhibitor (Boehringer Mannheim, Mannheim, Germany), treated with lysozyme DNase and RNase on ice, and centrifuged to remove cell debris. The endo $\mathrm{N}$ was absorbed to a column of Ni-NTA-coupled Superflow resin (Qiagen) and eluted with lysate buffer containing $250 \mathrm{~mm}$ imidazole. Fractions containing endo $\mathrm{N}$, as determined by SDS-PAGE, were diluted with an equal volume of glycerol and dialyzed at $4^{\circ} \mathrm{C}$ against $\mathrm{PBS}, \mathrm{pH}$ 8.0, and $50 \%$ glycerol. The specific activity of the purified endo N (dilutions containing 2.5-160 ng) was determined using $1 \mu \mathrm{g}$ of PSA-NCAM substrate obtained from embryonic chick brain and a 30 min reaction at $37^{\circ} \mathrm{C}$. After the reaction, the protein was adsorbed to nitrocellulose and immunostained for residual PSA using the $5 \mathrm{~A} 5 \mathrm{IgM}$ monoclonal antiPSA and goat anti-mouse IgM coupled to alkaline phosphatase. One unit of activity was defined as the amount of enzyme required to remove 


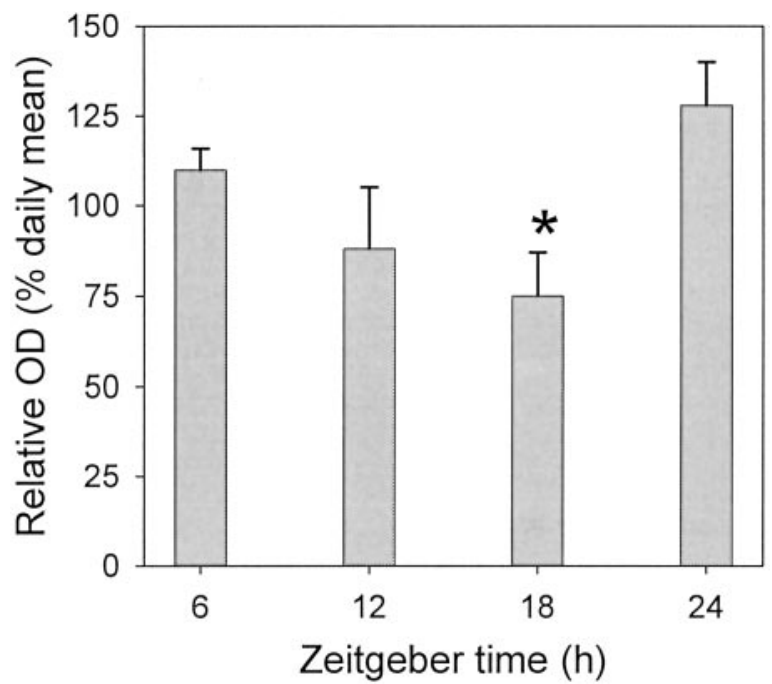

Figure 1. Circadian variation in the tissue content of PSA in the SCN at different times of the circadian cycle assessed by immunoblot assay. $0 D, 0$ ptic density. $n=3$ per time point. Error bars are SEM; ${ }^{*} p<0.05$ versus ZT 24 level.

one-half of the PSA staining. The purified endo $\mathrm{N}$ had no detectable protease activity or toxicity in $3 \mathrm{~d}$ cultures of primary neurons.

\section{Statistical analyses}

Differences in time of peak neuronal activity were assessed using Student's $t$ test or ANOVA analyses when appropriate. Differences in PSA expression were assessed by one-way ANOVA, followed by Tukey's multiple comparison post hoc test. In all cases, the level of significance was set at $p<0.05$.

\section{Results}

The circadian rhythm of SCN PSA expression persists in vitro PSA levels in the SCN slice preparation exhibited a distinct and reproducible rhythm, similar to that seen in vivo, with higher levels during the subjective day and lower levels at night (Fig. 1). The tissue content of PSA at ZT 18 was $75.0 \pm 0.1 \%$ of the daily mean ( $p<0.05$ vs ZT 24 level).

\section{endo $\mathrm{N}$ removes SCN PSA in vitro}

endo N selectively cleaves PSA from its NCAM carrier (Vimr et al., 1984; Acheson et al., 1991), and, for the following studies, it is critical that this treatment is effective with the SCN tissue slices. Using immunohistochemical and immunoblot procedures, it was found that the addition of endo $\mathrm{N}$ into the perfusion medium, as described below in the photic phase-shifting experiments, completely removed immunoreactive PSA from the SCN slice within $2 \mathrm{hr}$. Shown in Figure 2 are representative SCN slices, corresponding to subjective daytime, after immunostaining for PSA. Evident from the control slice is the strong pattern of PSA expression throughout the SCN, demarcating the nucleus from the surrounding hypothalamus and optic chiasm. In contrast, PSA staining was absent in the endo $\mathrm{N}$-treated slice. The endo $\mathrm{N}$ removal of PSA from the slice was confirmed by immunoblot assay (data not shown).

\section{Glutamate induces SCN PSA expression in vitro}

Having determined that PSA in the SCN in vitro is low during the subjective night, it was important to determine whether glutamate treatment affects PSA levels in the SCN at a time when glutamate has been shown to induce $3-4 \mathrm{hr}$ phase delays (ZT 14) (Ding et al., 1994; Forrest and Prosser, 2000). In the present
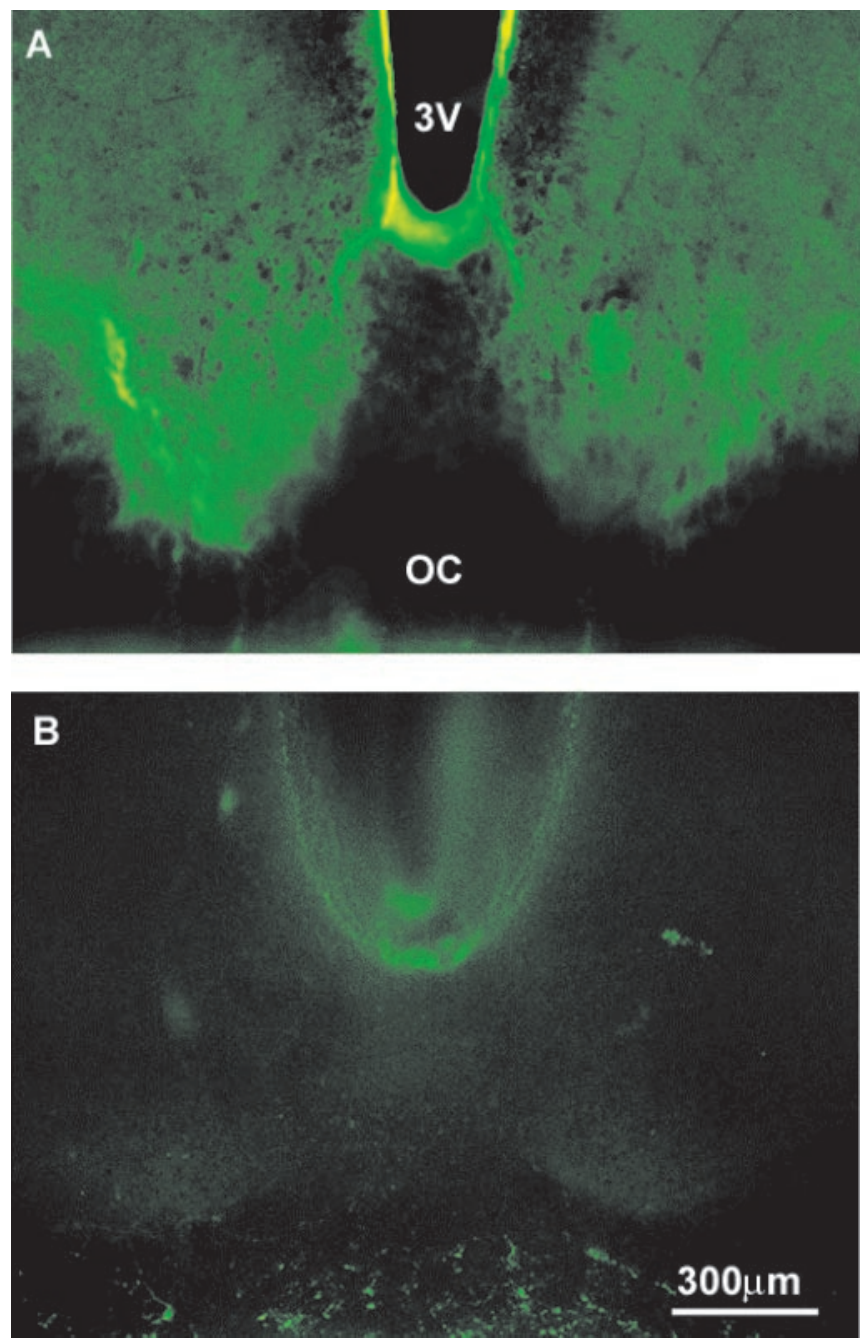

Figure 2. Immunohistochemical staining for PSA in the SCN at ZT 6. The PSA ( green labeling) is clearly seen throughout the $S C N$ under normal in vitro conditions $(A)$ but is absent from the $\mathrm{SCN}$ after treatment with endo $\mathrm{N}(B) .3 \mathrm{~V}$, Third ventricle; $O C$, optic chiasm.

experiments, slices treated for $10 \mathrm{~min}$ with glutamate $(1 \mathrm{mM})$ at ZT 14 showed a significant increase in PSA expression to $200 \%$ of the pretreatment level (Fig. 3) ( $p<0.05$ vs pretreatment level). This increase occurred within 30 min of the glutamate application and lasted $\sim 2 \mathrm{hr}$, after which PSA expression decreased to levels measured in untreated slices. Glutamate application at ZT 6 for $10 \mathrm{~min}$, when in vitro PSA levels are already high, similarly increased PSA levels $30 \mathrm{~min}$ later to $173 \pm 8 \%(n=3)$ of controls ( $p<0.01$ vs untreated slices). Conversely, a 10 min application of a mixture of glutamate antagonists (CNQX and AP-5) at ZT 6 did not significantly change PSA expression in vitro (data not shown).

\section{endo N blocks glutamate induction of SCN PSA in vitro}

Using the same procedure as in the glutamate experiment, SCN slices were exposed to endo $\mathrm{N}$ for $3 \mathrm{hr}$ beginning at ZT 12. At ZT 14 , they were given a $10 \mathrm{~min}$ pulse of glutamate $(1 \mathrm{mM})$. As shown in Figure 3, with endo N present, no PSA was detectable from ZT 14 to ZT 17, confirming that the concentration of enzyme used in these trials is sufficient to block the stimulated increase in PSA that occurs in response to a potentially phase-resetting application of glutamate. 


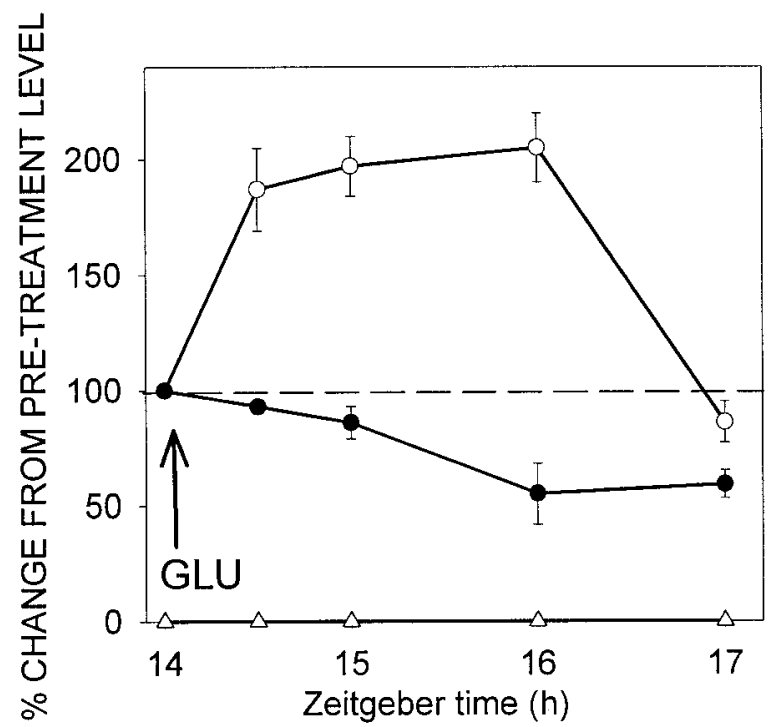

Figure 3. Glutamate acutely stimulates PSA expression in the $S C N$ slice. A 10 min application of glutamate induced an $\sim 200 \%$ increase in total SCN PSA content within 30 min of treatment. The PSA levels in untreated controls exhibited a gradual decrease throughout the subjective night. Pretreatment of slices with endo N prevented any glutamate-induced stimulation of PSA. Each time point is an average of three samples. Open circles, Glutamate-treated tissue; filled circles, no treatment; open triangles, glutamate plus endo $\mathrm{N}$-treated tissue.

endo $\mathrm{N}$ blocks phase delays induced by in vitro electrical stimulation of the optic chiasm

Electrical stimulation of the optic chiasm (OCS) was used to directly confirm an essential role for PSA in photic-like phaseresetting events in the SCN slice. The OCS procedure mimics photic activation of the SCN and induces phase shifts in vitro that are similar in magnitude and direction to those induced by glutamate (Ding et al., 1994; Forrest and Prosser, 2000). This is believed to occur because electrical stimulation induces release of the retinohypothalamic neurotransmitter glutamate from the retinal ganglion cell terminals (Liou et al., 1986). Depicted in Figure $4, A$ and $B$, are representative experiments showing the neuronal activity rhythm from an untreated slice and a $3.5 \mathrm{hr}$ phase delay in the neuronal activity rhythm induced by $10 \mathrm{~min}$ of OCS at ZT 14. When OCS was combined with $3 \mathrm{hr}$ endo $\mathrm{N}$ treatment from ZT 12 to ZT 15, the phase delays were completely blocked (Fig. 4C). In contrast, OCS applied to SCN slices in the presence of inactive (boiled) endo $\mathrm{N}$ continued to induce robust phase delays (Fig. $4 \mathrm{D}$ ). Slices receiving a $3 \mathrm{hr}$ incubation with endo $\mathrm{N}$ alone showed no phase-resetting response (data not shown). The results of these experiments are summarized graphically in Figure 5.

\section{endo $\mathrm{N}$ blocks glutamate-induced phase delays in vitro}

In a complementary experiment, the effects of endo $\mathrm{N}$ on the phase-resetting action of the RHT transmitter glutamate were assessed. This approach was also used to explore a post-RHT mechanism of action of PSA. Depicted in Figure $6 A$ is a representative experiment showing the phase-delaying effect of a 10 min pulse of glutamate delivered to the slice at ZT 14. As with OCS, this treatment induced a robust phase delay of $3.5 \mathrm{hr}$ in the circadian rhythm of neuronal activity compared with untreated slices. Conversely, in slices incubated with endo $\mathrm{N}$ for $3 \mathrm{hr}$ from ZT 12 to ZT 15 , the phase-resetting effect of glutamate is totally abolished (Fig. 6B). These results are summarized graphically in Figure 5.

\section{Discussion}

The demonstration that PSA expression in the SCN slice fluctuates with a similar pattern as that observed in vivo (Glass et al., 2001) attests to the SCN slice being a physiologically viable model for studying PSA function in the circadian clock. Using this system, the present results point to an essential role for PSA-NCAM in photic regulation of the mammalian circadian clock. Notably, when PSA is enzymatically removed from the SCN slice, the phase-resetting effects of photic-like signaling stimuli (electrical stimulation of the optic chiasm and application of glutamate) are blocked. This functional link between PSA and photic entrainment is consistent with the correlative observation that glutamate-induced phase delays occur in conjunction with a dramatic upregulation of SCN PSA expression. These results support previous in vivo studies pointing to a role for PSA in photic entrainment (Shen et al., 1997; Glass et al., 2000b). Most importantly, because removing PSA blocks the direct action of glutamate in the SCN, PSA must act downstream from presynaptic RHT terminals and therefore is intrinsic to SCN function.

Pattern and kinetics of PSA expression in the SCN slice Analyses of PSA immunostaining in the SCN of a number of species, including mouse and Siberian and Syrian hamsters, have revealed that PSA expression occurs throughout the rostrocaudal extent of the SCN, with denser staining in the ventrolateral ("core") compared with the dorsomedial ("shell") region (Glass et al., 1994, 2001; Shen et al., 1997). Whether the more intense staining in the core region reflects an association between PSA and retinorecipient units, which are found predominantly in the ventrolateral region (Morin, 1994), or whether it is a consequence of the higher cell density in this part of the SCN (van den Pol, 1980) is not known. However, in the hamster, intense PSA immunoreactivity is closely associated with cells expressing lightinduced Fos protein and with calbindin $\mathrm{D}_{128 \mathrm{~K}}$ cells, which receive RHT input (Bryant et al., 2000; Glass et al., 2001). From the present immunohistochemical analysis of vibratome sections of rat SCN slices, the pattern of PSA expression is similar to that observed in the SCN of the other rodent species, indicative of a similar generalized association between PSA-expressing elements and retinorecipient units.

Within the SCN, PSA expression is regulated by the circadian pacemaker: under constant dark conditions, PSA levels peak during subjective day and are lowest during subjective night in vivo (Glass et al., 2001). Here we show that a similar pattern is expressed virtually unchanged in vitro. This indicates that circadian control of PSA expression involves processes intrinsic to the SCN and does not rely on clock outputs indirectly feeding back onto the SCN.

Expression of PSA in the SCN is also strongly influenced by photic input, because light pulses presented to hamsters during late subjective night rapidly increase the normally low SCN PSA content to high daytime levels, as assessed using quantitative immunoblot analysis (Glass et al., 2000a). This response was specific to the SCN, because PSA levels in other regions were unaffected by light. Here we show that photic-like stimulation also increases PSA expression in the SCN in vitro using the same immunoblot analysis. In these trials, application of glutamate at ZT 14 increased PSA levels with a time course remarkably similar to that seen in vivo [i.e., PSA content increased within $30 \mathrm{~min}$ of stimulation in both cases and remained elevated for $1 \mathrm{hr}$ (in vivo) or 2 $\mathrm{hr}$ (in vitro) before returning to unstimulated levels]. Interestingly, glutamate applied during the subjective day increased PSA levels to a comparable degree. The fact that PSA content of the 

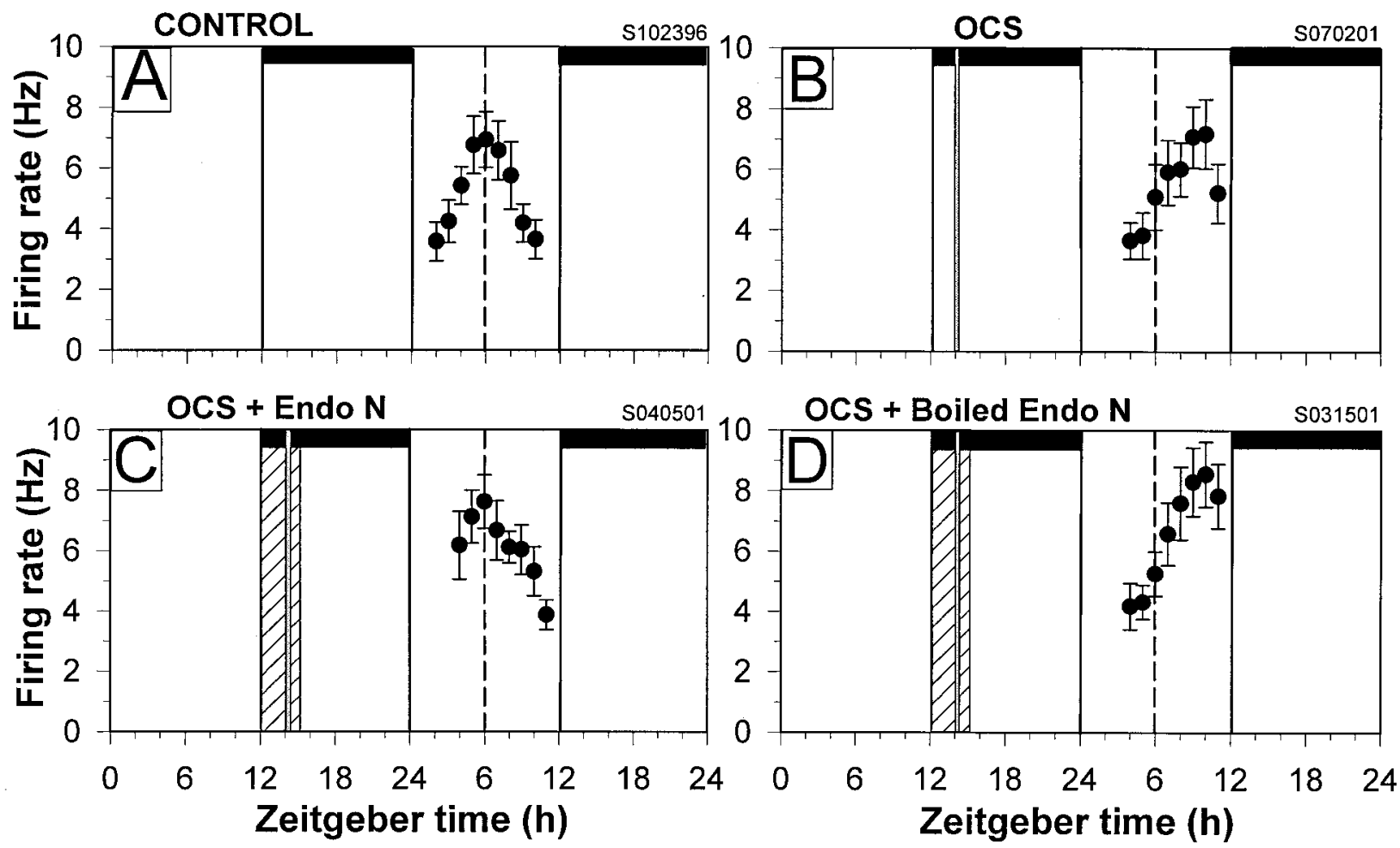

Figure 4. endo $\mathrm{N}$ blocks in vitro phase shifts induced by optic chiasm stimulation. Shown are representative single-unit activity recordings from SCN slices after the following treatments: $A$, no treatment, showing the normal peak in activity at ZT 6; $B$, optic chiasm stimulation at ZT 14 , showing a 3.5 hr phase delay in activity; $C$, optic chiasm stimulation plus endo N, showing no phase shift; and D, optic chiasm stimulation plus inactive endo N, showing a $3.5 \mathrm{hr}$ phase delay. Horizontal bars, Time of lights-off in the animal colony; vertical bars, times of treatment; dotted lines, mean time of peak neuronal activity in control slices.

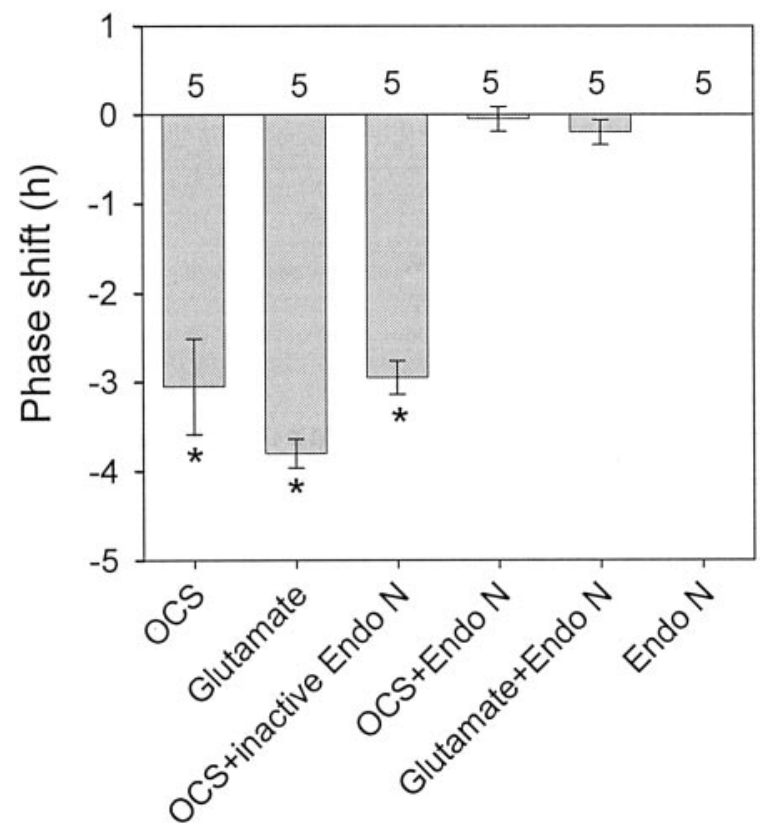

Figure 5. Histogram plot summarizing the results of phase-shifting experiments. Shown are the mean \pm SEM phase shifts induced by the different experimental conditions relative to the mean time-of-peak in control slices (ZT $6.0 \pm 0.3 ; n=3)$. Numbers below each bar indicate the number of replicate experiments. ${ }^{*} p<0.05$ versus untreated slices.

SCN increases after glutamate application in vitro provides strong evidence that the processes mediating photic induction of PSA are contained within the SCN. The rapid time course for the increase in PSA content suggests further that the increase in SCN
PSA is the result of a post-transcriptional event in PSA synthesis, most likely a change in the activity of polysialyltransferase, the enzyme that catalyzes PSA synthesis and attachment to its NCAM carrier. Such a change in activation has in fact been observed in the response of neurons when they establish connections with their targets (Bruses and Rutishauser, 1988). Our results showing daytime as well as nighttime increases in PSA levels in response to glutamate, coupled with the inability of glutamate antagonists to decrease daytime PSA expression, suggests that the in vitro rhythm in PSA expression is not being driven by a rhythm in glutamate release in this deafferented preparation. Thus, at least in vitro, endogenous control of PSA expression must involve nonglutamatergic mechanisms.

\section{PSA and photic phase resetting}

With respect to cellular mechanisms, the most salient finding of this study was that transient removal of PSA from the SCN completely blocked the phase-shifting effects of glutamate. This blockade indicates that the effects of PSA occur downstream from the presynaptic RHT terminals; that is, they are central to the regulation of the SCN clock rather than being derivative of effects upstream from the receipt of photic information. This is a fundamental distinction, because PSA is known to have a variety of effects on axon sprouting and synaptogenesis in CNS target regions (Bruses and Rutishauser, 2001). It is noteworthy that daytime glutamate induction of PSA (shown here) is not accompanied by pacemaker resetting (Ding et al., 1994). This indicates that additional processes characteristic of the nocturnal SCN must also be needed for photic phase shifting to occur.

The involvement of PSA in fundamental nervous system physiology has been noted in other studies. For example, PSA is 

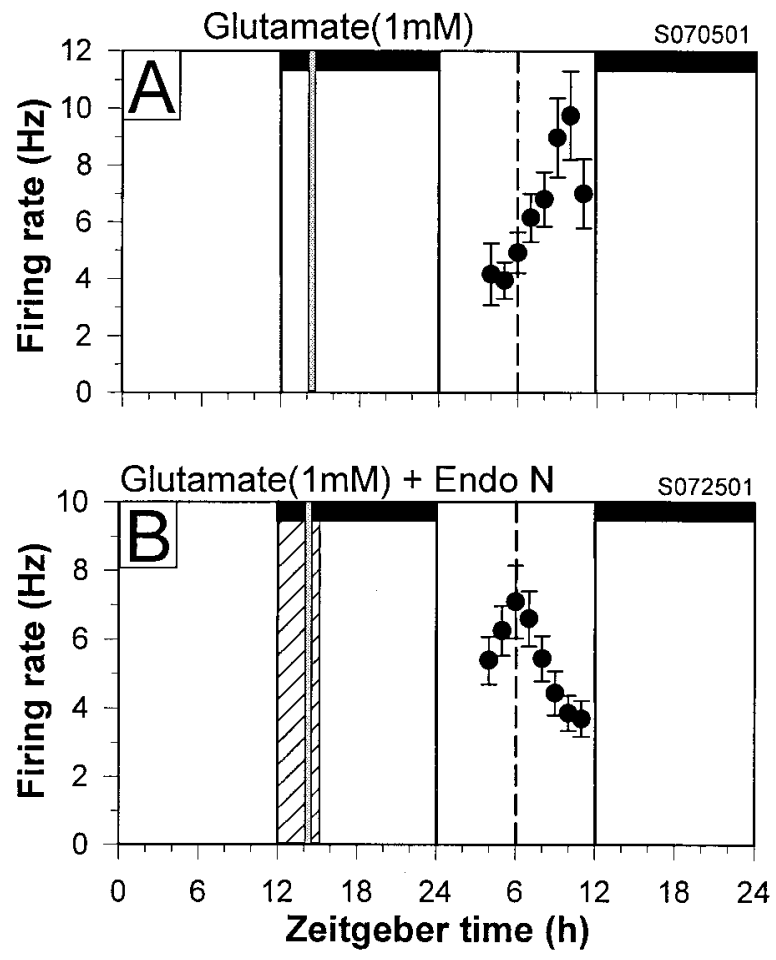

Figure 6. endo N blocks in vitro phase shifts induced by glutamate application to the SCN. Shown are representative single-unit activity recordings from SCN slices after the following treatments: $A$, glutamate (1 mm) at ZT14, showing a $3.5 \mathrm{hr}$ delay in neuronal activity; and $B$, glutamate (1 mM) plus endo N, showing no phase shift. For details, see Figure 4 legend.

essential for normal hypothalamo-neurohypophyseal activities during lactation and dehydration (Theodosis et al., 1999), and there is a correlation between PSA expression and LTP-associated morphological reorganizations of adult hippocampus (Regan and Fox, 1995; Muller et al., 1996; Cremer et al., 2000; Ronn et al., 2000). With respect to synaptic mechanisms, it is notable that the SCN exhibits LTP and long-term depression (Nishikawa et al., 1995; van den Pol et al., 1996), and we showed that some PSANCAM is also closely associated with synaptic complexes in the SCN (Shen et al., 1999). The cellular mechanisms proposed for PSA involvement in these two systems are very different, namely modulating synaptic efficacy of CA1 neurons versus facilitating changes in neuronal-glial cell interactions that control hormone release. Interestingly, either of these mechanisms could be relevant to the SCN, in which changes in both neuronal connectivity and glial cell influences have been noted (Lavialle and Serviere, 1993; Colwell, 2000; Harley et al., 2001).

Other studies on the role of PSA in learning and memory suggest that it may enhance the ability of the neurotrophin brainderived neurotrophic factor (BDNF) to interact with its $\operatorname{Trk} \beta$ receptor. Electrically stimulated hippocampal slices from PSANCAM knock-out mice and hippocampal slices treated with endo $\mathrm{N}$ exhibit reduced amounts of $\operatorname{Trk} \beta$ phosphorylation, which is an indicator of BDNF activity. Notably, treatment with exogenous BDNF reverses the loss of LTP and decreases $\operatorname{Trk} \beta$ phosphorylation exhibited by these slices (Muller et al., 2000). A similar interaction between PSA and BDNF has been seen with respect to cortical cell differentiation (Vutskits et al., 2001). Although it is not known whether PSA enhances BDNF activity in the SCN, it is noteworthy that recent data point to a role for BDNF in regulating photic shifting of the SCN clock (Liang et al., 1998, 2000).
Although removing PSA blocks the effects of glutamate, slices treated with endo $\mathrm{N}$ retain their normal circadian cyclicity, as assessed the day after enzyme treatment, with no significant change in rhythm phase or amplitude. Therefore, endo $\mathrm{N}$ treatment is not injurious to the neural clock apparatus, and removing PSA, at least temporarily, does not interfere with ongoing pacemaker activity. This latter observation indicates that the role of PSA-NCAM in the SCN may be restricted to clock entrainment and not to pacemaker signal generation per se.

In other studies, we found that PSA also influences nonphotic responses of the SCN (Fedorkova et al., 2002). Together with the present findings, this suggests that PSA expression in the SCN is requisite for all phase-shifting responses of the circadian clock (both photic and nonphotic) and therefore represents a central component of clock plasticity. Such a role, however, will likely prove to be complex, in that the nonphotic phase-resetting effect of the serotonin agonist 8 -hydroxy-2(di- $n$-propylamino)tetralin is significantly enhanced, rather than blocked, by endo $\mathrm{N}$ treatment (Fedorkova et al., 2002). One explanation for these opposite effects is that PSA enhances the response to glutamate, and, because glutamate is known to block serotonergic phase shifts in vitro (Prosser, 2001), removal of PSA could in principle reduce the glutamatergic inhibition of serotonergic phase-resetting actions. As a consequence, high levels of PSA during the day may normally act as a physiological dampener of daytime phaseresetting effects of nonphotic stimuli.

In conclusion, PSA expression in the acute SCN slice is regulated by the circadian pacemaker and by the photic-like influence of glutamate. Furthermore, enzymatic removal of PSA from the isolated SCN blocks the phase-resetting effects of glutamate and optic chiasm stimulation. Collectively, these data point to a critical role for PSA in the photic regulation of mammalian circadian clock phase.

\section{References}

Acheson A, Sunshine JL, Rutishauser U (1991) NCAM polysialic acid can regulate both cell-cell and cell-surface interactions. J Cell Biol 114:143-153.

Becker CG, Artola A, Gerardy-Schahn R, Becker T, Welzl H, Schachner M (1996) The polysialic acid modification of the neural cell adhesion molecule is involved in spatial learning and hippocampal long-term potentiation. J Neurosci Res 45:143-152.

Bonfanti L, Olive S, Poulain DA, Theodosis DT (1992) Mapping of the distribution of polysialylated neural cell adhesion molecule throughout the central nervous system of the adult rat: an immunohistochemical study. Neuroscience 49:419-436.

Bruses JL, Rutishauser U (1998) Regulation of NCAM polysialylation: evidence for nontranscriptional control and sensitivity to an intracellular pool of calcium. J Cell Biol 140:1177-1186.

Bruses JL, Rutishauser U (2001) Roles, regulation, and mechanism of polysialic acid function during neural development. Biochimie 83:635-643.

Bryant DN, LeSauter J, Silver R, Romero M-T (2000) Retinal innervation of calbindin- $\mathrm{D}_{28 \mathrm{~K}}$ cells in the hamster suprachiasmatic nucleus: ultrastructural characterization. J Biol Rhythms 15:103-111.

Chuong CM, Edelman GM (1984) Alterations in neural cell adhesion molecules during development of different regions of the nervous system. J Neurosci 4:2354-2368.

Colwell CS (2000) Rhythmic coupling among cells in the suprachiasmatic nucleus. J Neurobiol 43:379-388.

Cremer H, Chazal G, Lledo PM, Rougon G, Montaron MF, Mayo W, Le Moal M, Abrous DN (2000) PSA-NCAM: an important regulator of hippocampal plasticity. Int J Dev Neurosci 18:213-220.

Ding JM, Chen D, Weber ET, Faiman LE, Rea MA, Gillette MU (1994) Resetting the biological clock: mediation of nocturnal circadian shifts by glutamate and NO. Science 266:1713-1717.

Fedorkova L, Rutishauser U, Prosser RA, Shen H, Glass JB (2002) Removal 
of polysialic acid from the SCN potentiates nonphotic circadian phase resetting. Physiol Behav 77:361-369.

Forrest JB, Prosser RA (2000) Interactions between glutamate and serotonin agonists in phase-shifting the mammalian circadian clock in vitro at night. Soc Neurosci Abstr 26:76.2.

Fujimoto I, Bruses JL, Rutishauser U (2001) Regulation of cell adhesion by polysialic acid. J Biol Chem 276:31745-31751.

Gillette MU, Tischkau SA (1999) Suprachiasmatic nucleus: the brain's circadian clock. Rec Prog Horm Res 54:33-59.

Gillette MU, Medanic M, McArthur AJ, Liu C, Ding JM, Faiman LE, Weber ET, Tcheng TK, Gallman EA (1995) Intrinsic neuronal rhythms in the suprachiasmatic nuclei and their adjustment. CIBA Found Symp 183:134-153.

Glass JD, Lee W, Shen H, Watanabe M (1994) Expression of immunoreactive polysialylated neural cell adhesion molecule in the suprachiasmatic nucleus. Neuroendocrinology 60:87-95.

Glass JD, Fedorkova L, Chen L (2000a) Acute induction of polysialic acid in the SCN by light: neural plasticity and signal transduction in the hamster circadian clock. Soc Neurosci Abstr 26:304.8.

Glass JD, Shen H, Fedorkova L, Chen L, Tomasiewicz H, Watanabe M (2000b) Polysialylated neural cell adhesion molecule modulates photic signaling in the mouse suprachiasmatic nucleus. Neurosci Lett 280:207-210

Glass JD, Watanabe M, Fedorkova L, Shen H, Rutishauser U (2001) Dynamic expression of PSA-NCAM in a subregion of the hamster suprachiasmatic nucleus. Soc Neurosci Abstr 27:182.10.

Harley CW, Farrell RC, Rusak B (2001) Daily variation in the distribution of glycogen phosphorylase in the suprachiasmatic nucleus of Syrian hamsters. J Comp Neurol 435:249-258.

Hatton GI, Doran AD, Salm AK, Tweedle CD (1980) Brain slice preparation: hypothalamus. Brain Res Bull 5:405-414.

Lavialle M, Serviere J (1993) Circadian fluctuations in GFAP distribution in the Syrian hamster suprachiasmatic nucleus. NeuroReport 4:1243-1246.

Le Gal La Salle G, Rougon G, Valin A (1992) The embryonic form of neural cell surface molecule (E-NCAM) in the rat hippocampus and its reexpression on glial cells following kainic acid-induced status epilepticus. J Neurosci 12:872-882.

Lee W, Watanabe M, Glass JD (1995) Photoperiod affects the expression of neural cell adhesion molecule and polysialic acid in the hypothalamus of the Siberian hamster. Brain Res 690:64-72.

Liang F-Q, Walline R, Earnest DJ (1998) Circadian rhythm of brain-derived neurotrophic factor in the rat suprachiasmatic nucleus. Neurosci Lett 242:89-92.

Liang F-Q, Allen G, Earnest D (2000) Role of brain-derived neurotrophic factor in the circadian regulation of the suprachiasmatic pacemaker by light. J Neurosci 20:2978-2987.

Liou SY, Shibata S, Iwasaki K, Ueki S (1986) Optic nerve stimulationinduced increase of release of ${ }^{3} \mathrm{H}$-glutamate and ${ }^{3} \mathrm{H}$-aspartate but not ${ }^{3} \mathrm{H}$-GABA from the suprachiasmatic nucleus in slices of rat hypothalamus. Brain Res Bull 16:527-531.

Miragall F, Kadmon G, Faissner A, Antonicek H, Schachner M (1990) Retention of J1/tenascin and the polysialylated form of the neural cell adhesion molecule (N-CAM) in the adult olfactory bulb. J Neurocytol 19:899-914.

Morin LP (1994) The circadian visual system. Brain Res Rev 67:102-127.

Muller D, Wang C, Skibo G, Toni N, Cremer H, Calaora V, Rougon G, Kiss JZ (1996) PSA-NCAM is required for activity-induced synaptic plasticity. Neuron 17:413-422.

Muller D, Djebbara-Hannas Z, Jourdain P, Vutskits L, Durbec P, Rougon G, Kiss JZ (2000) Brain-derived neurotrophic factor restores long-term potentiation in polysialic acid-neural cell adhesion molecule-deficient hippocampus. Proc Natl Acad Sci USA 97:4315-4320.

Murakami S, Seki T, Wakabayashi K, Arai Y (1991) The ontogeny of luteinizing hormone-releasing hormone (LHRH) producing neurons in the chick embryo: possible evidence for migrating LHRH neurons from the olfactory epithelium expressing a highly polysialylated neural cell adhesion molecule. Neurosci Res 12:421-431.

Nishikawa Y, Shibata S, Watanabe S (1995) Circadian changes in long-term potentiation of rat suprachiasmatic field potentials elicited by optic nerve stimulation in vitro. Brain Res 695:158-162.

Prosser RA (1998a) In vitro circadian rhythms of the mammalian suprachiasmatic nuclei: comparison of multiunit and singleunit neuronal activity recordings. J Biol Rhythms 13:30-38.

Prosser RA (1998b) Neuropeptide Y blocks serotonergic phase shifts of the suprachiasmatic circadian clock in vitro. Brain Res 808:31-41.

Prosser RA (2001) Glutamate blocks serotonergic phase advances of the mammalian circadian pacemaker through AMPA and NMDA receptors. J Neurosci 21:7815-7822.

Prosser RA, Gillette MU (1989) The mammalian circadian clock in the suprachiasmatic nuclei is reset in vitro by cAMP. J Neurosci 9:1073-1081.

Prosser RA, Dean RR, Edgar DM, Heller HC, Miller JD (1993) Serotonin and the mammalian circadian system. I. In vitro phase shifts by serotonergic agonists and antagonists. J Biol Rhythms 8:1-16.

Regan CM, Fox GB (1995) Polysialylation as a regulator of neural plasticity in rodent learning and aging. Neurochem Res 20:593-598.

Ronn LCB, Berezin V, Bock E (2000) The neural cell adhesion molecule in synaptic plasticity and ageing. Int J Dev Neurosci 18:193-199.

Rutishauser U, Landmesser L (1996) Polysialic acid in the vertebrate nervous system: a promoter of plasticity in cell-cell interactions. Trends Neurosci 19:422-427.

Seki T, Arai Y (1991) The persistent expression of a highly polysialylated NCAM in the dentate gyrus of the adult rat. Neurosci Res 12:503-513.

Seki T, Arai Y (1993) Distribution and possible roles of the highly polysialylated neural cell adhesion molecule (NCAM-H) in the developing and adult central nervous system. Neurosci Res 17:265-290.

Seki T, Rutishauser U (1998) Removal of PSA-NCAM induces aberrant mossy fiber innervation and ectopic synaptogenesis in the hippocampus. J Neurosci 18:3757-3766.

Shen H, Watanabe M, Tomasiewicz H, Rutishauser U, Magnuson T, Glass JD (1997) Role of neural cell adhesion molecule and polysialic acid in mouse circadian clock function. J Neurosci 17:5221-5229.

Shen H, Glass JD, Seki T, Watanabe M (1999) Ultrastructural analysis of polysialylated neural cell adhesion molecule in the suprachiasmatic nuclei of the adult mouse. Anat Rec 256:448-457.

Shen H, Watanabe M, Tomasiewicz H, Glass JD (2001) Genetic deletions of NCAM and PSA impair circadian function in the mouse. Physiol Behav 73:185-193.

Theodosis DT, Rougon G, Poulain DA (1991) Retention of embryonic features by an adult neuronal system capable of plasticity: polysialylated neural cell adhesion molecule in the hypothalamo-neurohypophysial system. Proc Natl Acad Sci USA 88:5494-5498.

Theodosis DT, Bonhomme R, Vitiello S, Rougon G, Poulain DA (1999) Cell surface expression of polysialic acid on NCAM is a prerequisite for activity-dependent morphological neuronal and glial plasticity. J Neurosci 19:10228-10236.

van den Pol AN (1980) The hypothalamic suprachiasmatic nucleus of rat: Intrinsic anatomy. J Comp Neurol 191:661-702.

van den Pol AN, Obrietan K, Chen G, Belousov AB (1996) Neuropeptide $\mathrm{y}$-mediated long-term depression of excitatory activity in suprachiasmatic nucleus neurons. J Neurosci 16:5883-5895.

Viguie C, Jansen HT, Glass JD, Watanabe M, Billings HJ, Coolen L, Lehman MN, Karsch FJ (2001) Potential for polysialylated form of neural cell adhesion molecule-mediated neuroplasticity within the gonadotropinreleasing hormone neurosecretory system of the ewe. Endocrinology 142:1317-1324.

Vimr ER, McCoy RD, Vollger HF, Wilkison NC, Troy FA (1984) Use of prokaryotic-derived probes to identify poly(sialic acid) in neonatal neuronal membranes. Proc Natl Acad Sci USA 81:1971-1975.

Vutskits L, Djebbara-Hannas Z, Zhang H, Paccaud J-P, Durbec P, Rougon G, Muller D, Kiss JZ (2001) PSA-NCAM modulates BDNF-dependent survival and differentiation of cortical neurons. Eur J Neurosci 13:1391-1402.

Zlomanczuk P, Schwartz WJ (1999) Cellular and molecular mechanisms of circadian rhythms in mammals. In: Neurobiology of sleep and circadian rhythms (Turek FW, Zee P, eds), pp 309-342. New York: Dekker. 